\title{
Circulating microRNAs associated with prediabetes and geographic location in Latinos
}

\author{
Elena Flowers ${ }^{1,2}$ (1) Juan-Daniel Ramírez-Mares ${ }^{3} \cdot$ Marion Velazquez-Villafaña $^{3} \cdot$ Ruben Rangel-Salazar $^{3}$. \\ Anatol Sucher $^{4} \cdot$ Alka M. Kanaya $^{5,6} \cdot$ Bradley E. Aouizerat $^{7} \cdot$ Maria Luisa Lazo de la Vega Monroy ${ }^{3}$
}

Received: 23 April 2020 / Accepted: 23 December 2020 / Published online: 11 February 2021

(C) The Author(s) 2021

\begin{abstract}
Background Globally, type 2 diabetes is highly prevalent in individuals of Latino ancestry. The reasons underlying this high prevalence are not well understood, but both genetic and lifestyle factors are contributors. Circulating microRNAs are readily detectable in blood and are promising biomarkers to characterize biological responses (i.e., changes in gene expression) to lifestyle factors. Prior studies identified relationships between circulating microRNAs and risk for type 2 diabetes, but Latinos have largely been under-represented in these study samples.

Aims/hypothesis The aim of this study was to assess for differences in expression levels of three candidate microRNAs (miR126, miR-146, miR-15) between individuals who had prediabetes compared to normal glycemic status and between individuals who self-identified with Latino ancestry in the United States (US) and native Mexicans living in or near Leon, Mexico.

Methods This was a cross-sectional study that included 45 Mexicans and 21 Latino participants from the US. Prediabetes was defined as fasting glucose 100-125 mg/dL or 2-h post-glucose challenge between 140 and $199 \mathrm{mg} / \mathrm{dL}$. Expression levels of microRNAs from plasma were measured by qPCR. Linear and logistic regression models were used to assess relationships between individual microRNAs and glycemic status or geographic site.

Results None of the three microRNAs was associated with risk for type 2 diabetes. MiR-146a and miR-15 were significantly lower in the study sample from Mexico compared to the US. There was a significant interaction between miR-146a and BMI associated with fasting blood glucose.

Conclusions/interpretation This study did not replicate in Latinos prior observations from other racial groups of associations between miR-126, miR-146a, and miR-15 and risk for type 2 diabetes. Future studies should consider other microRNAs related to different biological pathways as possible biomarkers for type 2 diabetes in Latinos.
\end{abstract}

Keywords microRNA $\cdot$ Diabetes $\cdot$ Fasting blood glucose $\cdot$ Biomarker

\section{Introduction}

Type 2 diabetes is highly prevalent in individuals of Latino ancestry in both native countries of origin and in immigrants to

Elena Flowers

elena.flowers@ucsf.edu

1 Department of Physiological Nursing, University of California, San Francisco, 2 Koret Way, \#605L, San Francisco, CA 94143-0610, USA

2 Institute for Human Genetics, University of California, San Francisco, San Francisco, USA

3 Medical Sciences Department, Health Sciences Division, University of Guanajuato, Guanajuato, Mexico other countries. The prevalence of type 2 diabetes in Latinos living in the United States (US) is $12.7 \%$ [1] and the prevalence of type 2 diabetes in Mexico is $14.8 \%$ [2]. Progression to type 2 diabetes occurs on a continuum, and even in the prediabetes

4 University of California, San Francisco, San Francisco, USA

5 Department of Medicine, University of California, San Francisco, San Francisco, USA

6 Department of Epidemiology and Biostatistics, University of California, San Francisco, San Francisco, USA

7 College of Dentistry, Bluestone Center for Clinical Research, New York University, New York, USA 
state, harmful complications begin to occur [3]. Genetic risk factors for type 2 diabetes are common in some individuals of Latino ancestry $[4,5]$. However, Latinos are characterized by highly heterogeneous genetic admixture [6], and genetic risk for type 2 diabetes between individuals who identify as Latino may vary considerably by geographic ancestry. Furthermore, environmental, social, and lifestyle factors are also important contributors to risk for type 2 diabetes [7]. The complex etiology of type 2 diabetes makes it hard to accurately identify which individuals are at greatest risk and the specific mechanisms underlying risk for a given individual or population.

MicroRNAs are short (i.e., 18-26 nucleotide) regulatory elements of translation of messenger RNAs to amino acids. Circulating microRNAs found in serum and plasma are easily measured in blood and are potential biomarkers for risk for development of type 2 diabetes, characterizing changes in expression levels prior to the onset of prediabetes or type 2 diabetes $[8,9]$. Because microRNAs capture both underlying genetic risk as well as responses to environmental, social, and lifestyle factors $[10,11]$, they may be useful as biomarkers in two ways. The first is improved identification of which individuals are at greatest risk for type 2 diabetes. The second is information about specific patterns of gene expression in individuals at risk for type 2 diabetes, which is of particular interest for this complex condition because gene expression is driven by interactions between underlying genetic predisposition and environmental and lifestyle factors.

Prior studies on microRNAs associated with risk for type 2 diabetes have primarily been focused on non-Hispanic white and Asian populations [12]. The purpose of this study was to assess relationships between circulating microRNAs and prediabetes in individuals of Latino ancestry. We selected three microRNAs (i.e., miR-126, miR-146a, miR-15) previously shown to be associated with risk for type 2 diabetes in other racial groups to assess in this study $[13,14]$. We evaluated differences in microRNA expression levels between individuals who selfidentified with Latino ancestry in the United States (US) and native Mexicans living in or near Leon, Mexico, and individuals who were prediabetic compared to normal glycemic status.

\section{Research design and methods}

\section{Recruitment}

This was a multi-center observational cross-sectional study carried out at two different research institutions in Mexico and the US.

\section{Mexico}

Participants of Mexican ancestry were recruited from primary care health centers that serve the general population from the city of Leon in Guanajuato, Mexico. Participants included were between 35 and 65 years old without a previous diagnosis of prediabetes. Participants presenting with fasting glucose of $100-125 \mathrm{mg} / \mathrm{dL}$ or 2-h glucose between 140 and $199 \mathrm{mg} /$ $\mathrm{dL}$ after an oral glucose tolerance test (OGTT) were categorized as having prediabetes $(n=36)$. Participants with fasting glucose $<100 \mathrm{mg} / \mathrm{dL}$ and 2-h OGTT $<140 \mathrm{mg} / \mathrm{dL}$ were categorized as having normal glucose tolerance $(n=30)$. Exclusion criteria included history of diabetes, hypertension, thyroid, hepatic, immune, neoplastic, or endocrine disorder; statin, glucocorticoid, or anticonvulsant use; current smoking; and $>2$ drinks of alcohol/day.

\section{United States}

The US study sample included participants from the previously completed Practicing Restorative Yoga Metabolic Syndrome (PRYSMS) study (clinicaltrials.gov identifier NCT01024816), which tested the effects of restorative yoga versus active stretching on blood glucose in adults at risk for type 2 diabetes. Participants in the PRYSMS study were recruited from the San Francisco and San Diego areas and met the International Diabetes Federation criteria for metabolic syndrome [15]. The subset of participants who self-identified as Latino were included in this study $(n=21)$. Exclusion criteria included fasting glucose $\geq 126 \mathrm{mg} / \mathrm{dL}, \mathrm{HbAlc} \geq 7.0 \%$, fasting triglycerides $\geq 300 \mathrm{mg} / \mathrm{dL}$, weight $\geq 400 \mathrm{lbs}$, chronic disease, and neurological conditions that limited mobility, hospitalization for coronary heart disease within the past 6 months, current pregnancy or lactation, history of bariatric surgery, substance abuse, and use of medications affecting metabolic factors. The PRYSMS trial and the study described in this paper were approved by the Institutional Review Board at the University of California, San Francisco. All participants provided informed consent to participate in the study.

\section{Data Collection}

\section{Mexico}

The enrollment visit at the Mexico site included a brief medical history and family history for diabetes using the American Diabetes Association criteria [16]. Physical activity was assessed using the Spanish version of the self-administered short International Physical Activity Questionnaire (IPAQ) forms [17]. Participant height and weight were measured with a standardized stadiometer (Seca) and scale (Tanita BC-536). Hip and waist girth were measured with an anthropometric tape (Lufkin), and body composition was assessed using a bioimpedance device (InBody). Blood glucose and lipid levels were measured from serum samples by colorimetric enzymatic assays (Spinreact). Insulin was measured from serum using an ELISA kit (ALPCO) as per the manufacturer's instructions. 
Hemoglobin Alc (HbA1c) was analyzed via chromatography with Labona Check equipment and reagents in plasma. Plasma was stored at $-80{ }^{\circ} \mathrm{C}$ at both locations until used.

\section{United States}

The full clinical data collection protocol for the PRYSMS trial has been reported previously [18]. Participant weight was measured on a standard balance beam scale and height using a stadiometer. Waist circumference was measured using a Gullick II tape spring-tension measure at the site of maximum circumference midway between the lower ribs and the anterior superior iliac spine. The mean of two waist circumference measurements was calculated. Blood glucose was measured using an automated analyzer with an immobilized enzyme biosensor (YSI 2300 STAT Plus, YSI Life Sciences, Yellow Sprints, OH). Total cholesterol, triglycerides, and HDLcholesterol were measured by enzymatic colorimetric methods (Quest Diagnostics, San Jose, CA), and LDLcholesterol was calculated using the Friedewald equation [19]. Blood used for banking of plasma was collected by venipuncture. Blood was collected into vacutainers containing the preservative EDTA, centrifuged at $4{ }^{\circ} \mathrm{C}$ to separate plasma from cellular blood components, and stored at $-80{ }^{\circ} \mathrm{C}$.

\section{MicroRNA quantitation}

Study personnel from the Mexico site were trained in the isolation and quantitation of microRNAs at the US site. Both sites employed the same study protocol for all assays. RNA was extracted from $200 \mu \mathrm{L}$ of plasma using the miRNeasy serum/ plasma kit (Qiagen). Purified RNA was converted to cDNA using the miScript II RT Kit (Qiagen) in $20 \mu \mathrm{L}$ reaction volumes using the miScript HiSpec buffer. Real-time quantitative polymerase chain reaction (qPCR) was used to assess relative expression of candidate microRNAs using the miScript kit (Qiagen). Experiments were carried out using a 384-well (US) or 96-well (Mexico) plate format on a Bio-Rad CFX real-time PCR machine using the manufacturer's recommended cycling conditions. A standard curve was constructed for each microRNA target using a series of five serial dilutions. Both sites obtained at least three replicates measures per sample for each microRNA target. MicroRNA expression levels were normalized using cel-miR-39 and the global geometric mean signal of all reliably detected microRNAs [20, 21], and relative expression levels were calculated using the $\Delta \Delta \mathrm{Ct}$ method [22].

\section{Statistical analysis}

Descriptive statistics and Student's $t$ test were used to evaluate demographic and clinical characteristics of participants between study sites and glycemic status (Stata version 13, College Station, TX). Pearson's correlation coefficients were used to determine relationships between fasting blood glucose and covariates that are continuous variables. Logistic regression models were used to determine whether individual microRNAs were associated with prediabetes compared to normal glucose tolerance. Logistic regression models were also used to determine whether individual microRNAs were associated with the study site with the US as the reference site. Linear regression models were used to determine whether individual microRNAs were associated with fasting blood glucose. For all regression models, unadjusted models were first created. Next, variables that were significantly associated with prediabetes or study site were included as covariates in adjusted models. Finally, we included interaction terms for covariates that were significantly associated with individual microRNAs.

\section{Results}

A total of 45 participants were enrolled in Leon, Mexico, and 21 participants from the US-based PRYSMS trial who selfidentified as Latino were included in the study. Participants from Mexico were younger ( $46 \pm 8$ years versus $51 \pm 7$ years, $p<0.05)$ and had lower BMI $\left(29.8 \pm 3.8 \mathrm{~kg} / \mathrm{m}^{2}\right.$ versus $35.9 \pm$ $\left.8.1 \mathrm{~kg} / \mathrm{m}^{2}, p<0.05\right)$ and weight $(81.8 \pm 11.4 \mathrm{~kg}$ versus $92.3 \pm$ $18.2 \mathrm{~kg}, p<0.05$ ) (Table 1). While there were no differences in fasting blood glucose, hemoglobin A1c was higher in Latinos from the US compared to Mexicans from Mexico $(6.0 \pm 0.3 \%$ versus $4.4 \pm 0.5 \%, p<0.001)$ (Table 1$)$. In a multivariate-adjusted logistic regression model, BMI but not age or sex was significantly lower in individuals from Mexico compared to the US (OR 0.82 (95\% CI 0.71, 0.94)).

In the full study sample, $60 \%(n=36)$ of participants had prediabetes compared to normal glucose tolerance (Table 2). There was no difference in the proportion with prediabetes by study site. A higher proportion of individuals with prediabetes were female $(83 \%(n=30)$ versus $57 \%(n=17), p<0.05)$ and had higher BMI $\left(34.4 \pm 6.0 \mathrm{~kg} / \mathrm{m}^{2}\right.$ versus $29.7 \pm 5.8 \mathrm{~kg} / \mathrm{m}^{2}, p$ $<0.05)$. Fasting blood glucose was higher in individuals with prediabetes $(109 \pm 8 \mathrm{mg} / \mathrm{dL}$ versus $89 \pm 8 \mathrm{mg} / \mathrm{dL}, p<0.05)$ but there were no differences in hemoglobin A1c. In a multivariate-adjusted logistic regression model, BMI, but not age or sex, was significantly associated with risk for prediabetes (OR 1.12 (95\% CI 1.00, 1.26)).

All three microRNAs were strongly significantly correlated with each other (Table 3). MiR-146a was significantly associated with BMI $\left(r^{2}=0.28, p<0.05\right)$. There were no other significant correlations between individual microRNAs and age, sex, or BMI.

The distribution of normalized (i.e., $\Delta \mathrm{Ct}$ ) expression for each microRNA by study site is shown in Fig. 1. In unadjusted logistic regression models, both miR-146a (OR 0.83 (95\% CI $0.67,0.99))$ and miR-15 (OR $0.79(0.65,0.97))$ were 
Table 1 Demographic and clinical characteristics by study site

\begin{tabular}{llll}
\hline$\%(n)$ or average \pm standard deviation & US Latinos $(n=21)$ & Mexican $(n=45)$ & $p$ value \\
\hline Age & $51 \pm 7$ & $46 \pm 8$ & $<0.05$ \\
Sex (male) & $24(5)$ & $31(14)$ & 0.54 \\
Body mass index $\left(\mathrm{kg} / \mathrm{m}^{2}\right)$ & $35.9 \pm 8.1$ & $29.8 \pm 3.8$ & $<0.05$ \\
Weight $(\mathrm{kg})$ & $92.3 \pm 18.2$ & $81.8 \pm 11.4$ & $<0.05$ \\
Fasting blood glucose $(\mathrm{mg} / \mathrm{dL})$ & $101 \pm 11$ & $99 \pm 14$ & 0.44 \\
Hemoglobin A1c $(\%)$ & $6.0 \pm 0.3$ & $4.4 \pm 0.5$ & $<0.001$ \\
Prediabetes $(\%)$ & $57(12)$ & $67(24)$ & 0.77 \\
Total cholesterol $(\mathrm{mg} / \mathrm{dL})$ & $216 \pm 44$ & $194 \pm 34$ & 0.06 \\
LDL-c $(\mathrm{mg} / \mathrm{dL})$ & $134 \pm 37$ & $120 \pm 30$ & 0.13 \\
HDL-c $(\mathrm{mg} / \mathrm{dL})$ & $47 \pm 10$ & $39 \pm 11$ & $<0.05$ \\
Triglycerides $(\mathrm{mg} / \mathrm{dL})$ & $176 \pm 68$ & $165 \pm 6$ & 0.58 \\
\hline
\end{tabular}

significantly decreased in individuals from the Mexico study site compared to the US site (Table 4). In a model adjusted for age and BMI, miR-15 remained significantly lower in individuals from Mexico compared to the US (OR 0.76, (95\% CI $0.60,0.97)$ ). When we further added hemoglobin A1c to the model, which was higher in participants from the US compared to Mexico, miR-15 was no longer significant. There was no interaction between miR-15 and hemoglobin A1c. There was a significant interaction between miR-146a and BMI in both unadjusted and age- and BMI-adjusted linear regression models for fasting blood glucose.

The distribution of normalized (i.e., $\Delta \mathrm{Ct}$ ) expression for each microRNA by glycemic status is shown in Fig. 2. In unadjusted and sex- and BMI-adjusted logistic regression models, no microRNAs were significantly associated with higher odds for prediabetes. In unadjusted and sex- and BMI-adjusted linear regression models, no microRNAs were significantly associated with fasting blood glucose. However, there was a significant interaction between miR-146a and $\mathrm{BMI}$ in a linear regression model for fasting blood glucose $(\beta=-0.16,95 \% \mathrm{CI}(-0.32,-0.01))$. The test for interaction was not significant for miR-126 and miR-15.

\section{Discussion}

Compared to prior studies focused on relationships between circulating microRNAs and risk for type 2 diabetes, we did not find significant associations between miR-126, miR-146a, or miR-15 and prediabetes. Prior studies were primarily conducted in European or Asian populations [12, 23, 24], whereas we studied Latinos. We did identify a significant association between miR-146a and BMI, which is a relationship that has previously been observed in several studies of Europeans [25]. We also identified differences in expression levels of miR$146 \mathrm{a}$ and miR-15 between individuals living in or near Leon, Mexico, compared with individuals living in the US who self-identified as Latino.

MiR-146a has previously been associated with risk for type 2 diabetes in numerous studies, including a meta-analysis [24, 26]. Inflammation is one of the potential mechanisms by which miR-146a is hypothesized to have an effect on risk for type 2 diabetes and related conditions [27, 28]. There is strong experimental evidence that biological pathways from the Kyoto Encyclopedia of Genes and Genomes (KEGG) are targeted by miR-146, including ones related to inflammation
Table 2 Demographic and clinical characteristics by glycemic status

\begin{tabular}{llll}
\hline & Normal Glucose Tolerance $(n=30)$ & Prediabetes $(n=36)$ & $p$ value \\
\hline Study site (Mexico) & $70(21)$ & $67(24)$ & 0.77 \\
Age (years) & $47 \pm 9$ & $48 \pm 8$ & 0.40 \\
Sex (male) & $43(13)$ & $17(6)$ & $<0.05$ \\
Body mass index $\left(\mathrm{kg} / \mathrm{m}^{2}\right)$ & $29.7 \pm 5.8$ & $34.4 \pm 6.0$ & $<0.05$ \\
Weight $(\mathrm{kg})$ & $81.2 \pm 13.6$ & $88.6 \pm 14.5$ & 0.05 \\
Fasting blood glucose (mg/dL) & $89 \pm 8$ & $109 \pm 8$ & $<0.001$ \\
Hemoglobin A1c $(\%)$ & $4.8 \pm 0.7$ & $5.1 \pm 0.9$ & 0.18 \\
Total cholesterol (mg/dL) & $201 \pm 35$ & $201 \pm 41$ & 0.96 \\
LDL-c (mg/dL) & $125 \pm 27$ & $123 \pm 37$ & 0.85 \\
HDL-c (mg/dL) & $41 \pm 9$ & $42 \pm 13$ & 0.67 \\
Triglycerides (mg/dL) & $170 \pm 82$ & $167 \pm 77$ & 0.86 \\
\hline
\end{tabular}


Table 3 Correlation coefficients between individual microRNAs and covariates

\begin{tabular}{llllllll}
\hline & $\begin{array}{l}\text { MiR- } \\
126\end{array}$ & $\begin{array}{l}\text { MiR- } \\
146 \mathrm{a}\end{array}$ & MiR-15 & Age & Sex & BMI & FBG \\
& MiR-126 & 1.0000 & & & & & \\
MiR-146a & 0.9538 & 1.0000 & & & & & \\
& $<0.001$ & & & & & & \\
MiR-15 & 0.7406 & 0.6792 & 1.0000 & & & & \\
& $<0.001$ & $<0.001$ & & & & & \\
Age & -0.1470 & -0.0997 & -0.0646 & 1.0000 & & & \\
& 0.2388 & 0.4258 & 0.6063 & & & & \\
Sex & -0.2197 & -0.1761 & -0.1401 & 0.3649 & 1.0000 & & \\
& 0.0763 & 0.1573 & 0.2620 & 0.0026 & & & \\
BMI & 0.1687 & 0.2815 & 0.0362 & 0.3245 & 0.2314 & 1.0000 & \\
& 0.1757 & 0.0220 & 0.7730 & 0.0078 & 0.0616 & & \\
FBG & 0.0971 & 0.1235 & 0.1928 & 0.0917 & 0.1446 & 0.3144 & 1.0000 \\
& 0.4379 & 0.3232 & 0.1208 & 0.4639 & 0.2466 & 0.0101 & \\
\hline
\end{tabular}

Italicized font indicates values that were statistically significant

$B M I$, body mass index; $F B G$, fasting blood glucose (e.g., nuclear factor- $\kappa \beta$ (NF-K $\beta$ ) signaling pathway, Toll-like receptor signaling pathway, tumor necrosis factor- $\alpha$ signaling pathway) [29]. Findings from prior studies have been inconsistent in terms of the direction of the association (i.e., increased versus decreased expression of miR-146a and increased risk for type 2 diabetes). These discrepancies may partly result from differences in the tissue source from which microRNAs were obtained (e.g., plasma versus peripheral blood mononuclear cells (PBMCs)) and cross-sectional study design, which does not allow for characterization of where an individual lies on the glycemic spectrum. Our study did not find any association between miR-146a and prediabetes, which may be attributed in part due to differences in study design, including the examples listed above [26]. Another possible explanation for our null findings is that prior studies were primarily focused on European and Asian populations [12]. One prior study of microRNAs and risk for type 2 diabetes included Mexicans [30]. MiR-146a was significantly decreased in individuals with type 2 diabetes compared to healthy controls and was significantly correlated with BMI [30]; however, microRNAs were obtained from PBMCs, whereas our study focused on circulating microRNAs from plasma. Another study identified decreased expression of miR-146a associated with type 2 diabetes in Ecuadoreans and correlations between miR-146a and inflammatory markers [31]. Both of these prior studies were crosssectional and included individuals with a diagnosis of type 2 diabetes, whereas our study focused on prediabetes. There are many physiological changes across the glycemic spectrum from normal glucose tolerance to impaired fasting glucose (i.e., prediabetes) to type 2 diabetes that are not captured in cross-sectional study designs that use only fasting blood glucose to assess glycemic status.
Table 4 Odds ratios for prediabetes and study site

\begin{tabular}{llll}
\hline & miR-126, OR (95\% CI) & miR-146a, OR (95\% CI) & miR-15, OR (95\% CI) \\
\hline $\begin{array}{l}\text { Prediabetes } \\
\text { Unadjusted }\end{array}$ & $1.03(0.88,1.22)$ & $1.02(0.89,1.19)$ & $1.05(0.92,1.21)$ \\
Adjusted* & $1.05(0.87,1.27)$ & $1.02(0.85,1.22)$ & $1.09(0.93,1.27)$ \\
Study site $^{\#}$ & & & \\
Unadjusted $^{\text {Adjusted }}$ & $0.86(0.70,1.05)$ & $0.83(0.67,0.99)$ & $0.79(0.65,0.97)$ \\
\hline
\end{tabular}

Italicized font indicates values that were statistically significant

* Models are adjusted for sex and BMI

${ }^{\#}$ United States is the reference site

${ }^{\$}$ Models are adjusted for age and BMI

$B M I$, body mass index; $C I$ confidence interval; $O R$, odds ratio 
Fig. 1 Distribution of normalized microRNA expression levels by study site. Box and whisker plots show maximum (upper horizontal line), 75th percentile (upper border of box), 50th percentile/ median (mid-line of box), 25th percentile (lower border of box), and minimum (lower horizontal line). Black dots represent outliers. MiR, microRNA; MX, Mexico; US, United States

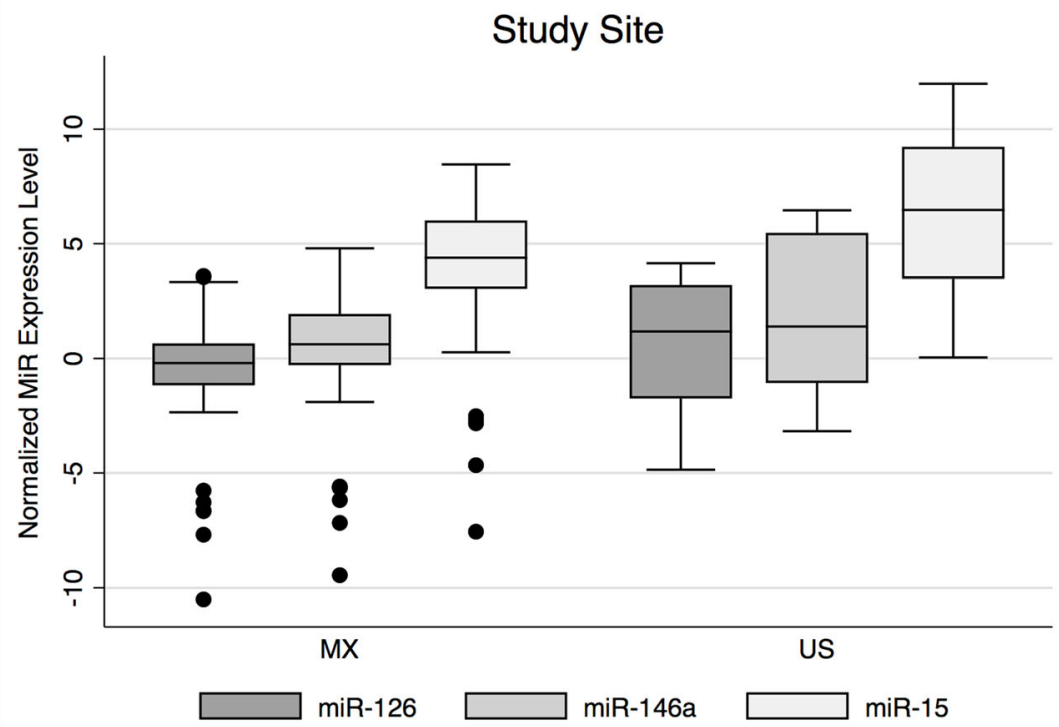

Overweight and obesity are major risk factors for type 2 diabetes, with approximately $40-70 \%$ of individuals at high risk for type 2 diabetes being overweight or obese [32]. We observed a significant correlation between miR-146a and BMI and a significant interaction between these two variables in determining odds for prediabetes versus normal glucose tolerance. MiR-146a has previously been associated with obesity in animal models [33, 34] and obesity-related inflammation in human adipocytes [35]. In human studies, miR-146a was increased in obese Chinese children and Chinese adults with type 2 diabetes and in functional in vitro and animal model studies increased miR-146a impaired $\beta$-cell function and insulin secretion [23]. Another KEGG pathway targeted by miR-146a is the adipocytokine signaling pathway. Overweight and obesity cause inflammation in part through the activity of adipocytokines, which are inflammatory molecules generated in adipose tissue [36-38]. The significant interaction that we observed between miR-146 and BMI suggests that the effect of BMI on prediabetes depends on the expression level of miR146a, or vice versa. The relationship between miR-146a and risk for type 2 diabetes may be linked to its effect on body composition and/or obesity-related inflammation. Future studies that include a longitudinal design, gold-standard assessments of body composition and glycemic status, and functional analysis of the impact of miR-146a on genes related to inflammation and obesity may further shed light on these relationships.

Fig. 2 Distribution of normalized microRNA expression levels by glycemic status. Box and whisker plots show 95th percentile (upper horizontal line), 75th percentile (upper border of box), 50th percentile/median (mid-line of box), 25th percentile (lower border of box), and minimum (lower horizontal line). Black dots represent outliers. MiR, microRNA; MX, Mexico; US, United States

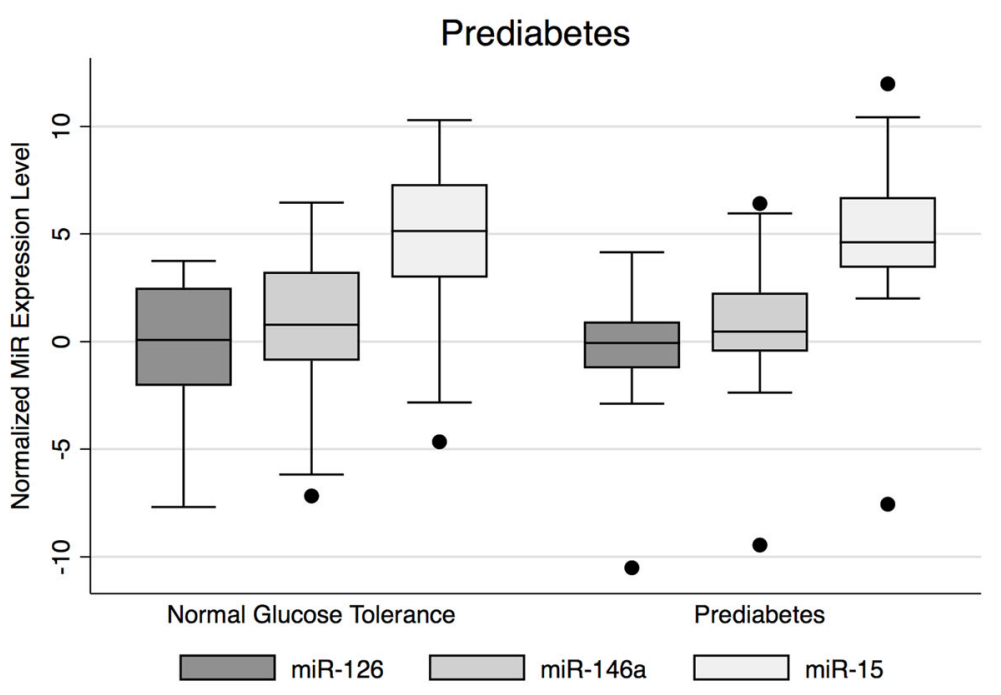


Prior studies identified associations between miR-15 and risk for type 2 diabetes [39, 40]. Baseline levels of miR-15 were lower in Spanish individuals who developed type 2 diabetes after 5 years, though miR-15 was not significantly associated with other measures of risk for type 2 diabetes (i.e., fasting blood glucose, hemoglobin A1c, measures of insulin sensitivity) [40]. A study of African-Americans identified a Ushaped curve in the relationship between miR-15 and the glycemic trajectory, with lower expression of miR-15 in individuals with prediabetes compared to individuals with normal fasting glucose or type 2 diabetes [39]. In the group with type 2 diabetes, miR-15 was associated with body weight and body mass index, but not hemoglobin A1c, and none of these associations was observed in the group with prediabetes [39]. In addition, miR-15 was able to discriminate between type 2 diabetes and prediabetes and between prediabetes and normal blood glucose, although these models were not compared to any other predictive or discriminatory models [39]. Our study showed that hemoglobin A1c, which differed between study sites (i.e., US versus Mexico) attenuated the relationship between miR-15 and study site. The relationship between miR15 and hemoglobin A1c remains relatively unstudied. Mechanistic studies of miR-15 identified regulation of NF- $K \beta[41,42]$ with corresponding increases in the inflammatory interleukin- 8 and interferon- $\gamma$ markers [42], suggesting that miR-15 may also contribute to regulation of inflammation observed in individuals at risk for type 2 diabetes.

MiR-126 was previously associated with risk for type 2 diabetes in numerous studies [14, 43]. Insights from mechanistic studies of miR-126 showed that this microRNA is associated with endothelial cell function [44-46], and therefore, differential expression may be associated with consequences from type 2 diabetes and elevated blood glucose levels [47]. Prior studies that identified associations between miR-126 and risk for type 2 diabetes focused on primarily European and Asian racial groups [43, 48-51]. Very little has been reported about microRNAs associated with risk for type 2 diabetes in Latino populations. Further studies are needed to validate our finding that miR-126 may not be associated with prediabetes in Latinos.

The Latino racial group category includes individuals from vast geographic regions with highly admixed genetic characteristics [6]. Characterization of individuals by this broad criterion may lack specificity about the degree of genetic similarity. For example, Latinos living in California have different genetic characteristics compared with Latinos living on the East Coast of the US or in Mexico and Central and South America [52]. Prior studies that included individuals of Latino origin (e.g., Ecuadorean) may have been genetically dissimilar to our study sample. In order to accurately assess genetic similarity between individuals from the Latino racial group, genetic admixture analysis is needed. Furthermore, behavioral and lifestyle factors that impact risk for type 2 diabetes vary considerably between individuals who are broadly categorized as Latino. Inconsistencies in the associations between individual microRNAs and risk factors for type 2 diabetes may be the result of not only differences between racial groups in terms of genetic admixture/ genetic risk and behavioral/lifestyle factors but also differences within racial groups (i.e., Latinos) that are unaccounted for by this very general categorization.

A limitation of this study and the majority of studies to date is the cross-sectional design. Development of type 2 diabetes occurs on a continuum, and cross-sectional studies fail to identify where on this continuum an individual may fall. Even within the clinically assigned categories of normal glucose tolerance, prediabetes, and type 2 diabetes, there may be differences in the underlying pathophysiology that impact expression levels of circulating microRNAs. Clinical and molecular data collection for this study was carried out at separate study sites. However, the laboratory protocols were identical at both sites, and study personnel were trained on the molecular data collection protocols at the US site. All data analysis was performed at a single site (US). There were some differences in demographic and clinical characteristics between the study sites, which were included as covariates in models. The genetic ancestry of all participants is not known, and the degree of genetic similarity between individuals is not known. Environmental and lifestyle factors were not assessed and conclusions about the impact of these potential risk factors on the associations between individual microRNAs and prediabetes cannot be made.

Circulating microRNAs are emerging as promising biomarkers for risk for type 2 diabetes. However, the majority of studies to date have primarily included individuals who identify as non-Hispanic white/European or Asian. Latinos have a particularly high prevalence of type 2 diabetes, and the reasons for this are not well understood. Given that circulating microRNAs capture the combined impact of genetic predisposition with responses to environmental and lifestyle factors, they may provide new insights about the reasons for increased risk for type 2 diabetes in some individuals and populations.

Funding This material is based upon work supported by a grant from the University of California Institute for Mexico and the United States (UC MEXUS) and the Consejo Nacional de Ciencia y Tecnología de México (CONACYT). Dr. Flowers was supported by the National Center for Advancing Translational Sciences of the National Institutes of Health grant number KL2TR000143 and the Hellman Family Foundation. Juan-Daniel Ramírez-Mares was the recipient of a scholarship from CONACYT (CVU: 708016). The PRYSMS study was supported by the National Center for Complementary and Alternative Medicine of the National Institutes of Health grant number 5R01AT004569. 


\section{Compliance with ethical standards}

Our research was approved by the University of California, San Francisco, and Institutional Review Boards and all procedures followed were in accordance with the ethical standards of the IRBs and the Helsinki Declaration of 1975, as revised in 2000.

Conflict of interest The authors do not have any relevant disclosures.

Ethical considerations The study was approved by the Institutional Committee of Bioethics of the University of Guanajuato (CIBIUG-P052016) and by the Research Committee of the VII Health Jurisdiction of the Health Secretary of Guanajuato (JS7-02-280217). All procedures were performed according to the Mexican General Health Laws and the Declaration of Helsinki. All participants provided informed consent to participate in the study.

Informed consent was obtained from all participants included in the study.

Open Access This article is licensed under a Creative Commons Attribution 4.0 International License, which permits use, sharing, adaptation, distribution and reproduction in any medium or format, as long as you give appropriate credit to the original author(s) and the source, provide a link to the Creative Commons licence, and indicate if changes were made. The images or other third party material in this article are included in the article's Creative Commons licence, unless indicated otherwise in a credit line to the material. If material is not included in the article's Creative Commons licence and your intended use is not permitted by statutory regulation or exceeds the permitted use, you will need to obtain permission directly from the copyright holder. To view a copy of this licence, visit http://creativecommons.org/licenses/by/4.0/.

\section{References}

1. Prevention, C.f.D.C.a. National Diabetes Statistics Report 2017, C.f.D.C.a. Prevention, Editor. 2017.

2. Federation, I.D., IDF North America and Caribbean members. 2017.

3. Brannick B, Wynn A, Dagogo-Jack S. Prediabetes as a toxic environment for the initiation of microvascular and macrovascular complications. Exp Biol Med (Maywood). 2016;241(12):1323-31.

4. Williams AL, et al. Sequence variants in SLC16A11 are a common risk factor for type 2 diabetes in Mexico. Nature. 2014;506(7486): 97-101.

5. Estrada K, et al. Association of a low-frequency variant in HNF1A with type 2 diabetes in a Latino population. JAMA. 2014;311(22): 2305-14.

6. Bryc K, Velez C, Karafet T, Moreno-Estrada A, Reynolds A, Auton $\mathrm{A}$, et al. Colloquium paper: genome-wide patterns of population structure and admixture among Hispanic/Latino populations. Proc Natl Acad Sci U S A. 2010;107(Suppl 2):8954-61.

7. González-Villalpando C, Dávila-Cervantes CA, Zamora-Macorra M, Trejo-Valdivia B, González-Villalpando ME. Risk factors associated to diabetes in Mexican population and phenotype of the individuals who will convert to diabetes. Salud Publica Mex. 2014;56(4):317-22.

8. Flowers E, Aouizerat BE, Abbasi F, Lamendola C, Grove KM, Fukuoka Y, et al. Circulating microRNA-320a and microRNA486 predict thiazolidinedione response: moving towards precision health for diabetes prevention. Metabolism. 2015;64:1051-9.
9. Flowers E, et al. Circulating micrornas associated with glycemic impairment and progression in Asian Indians. Biomarker Res. 2015;3(1):1-8.

10. Flowers E, Won GY, Fukuoka Y. MicroRNAs associated with exercise and diet: a systematic review. Physiol Genomics. 2015;47(1):1-11.

11. Parr EB, Camera DM, Burke LM, Phillips SM, Coffey VG, Hawley JA. Circulating microRNA responses between 'high' and 'low' responders to a $16-\mathrm{Wk}$ diet and exercise weight loss intervention. PLoS One. 2016;11:e0152545.

12. Zhu H, Leung SW. Identification of microRNA biomarkers in type 2 diabetes: a meta-analysis of controlled profiling studies. Diabetologia. 2015;58:900-11.

13. Raffort J, Hinault C, Dumortier O, van Obberghen E. Circulating microRNAs and diabetes: potential applications in medical practice. Diabetologia. 2015;58(9):1978-92.

14. Zampetaki A, Kiechl S, Drozdov I, Willeit P, Mayr U, Prokopi M, et al. Plasma microRNA profiling reveals loss of endothelial miR126 and other microRNAs in type 2 diabetes. Circ Res. 2010;107(6):810-7.

15. Alberti KG, Eckel RH, Grundy SM, Zimmet PZ, Cleeman JI, Donato KA, et al. Harmonizing the metabolic syndrome: a joint interim statement of the International Diabetes Federation Task Force on Epidemiology and Prevention; National Heart, Lung, and Blood Institute; American Heart Association; World Heart Federation; International Atherosclerosis Society; and international association for the Study of Obesity. Circulation. 2009;120(16): $1640-5$.

16. Association, A.D. 2. Classification and diagnosis of diabetes. Diabetes Care. 2018;41(Suppl 1):S13-27.

17. Craig CL, et al. International physical activity questionnaire: 12country reliability and validity. Med Sci Sports Exerc. 2003;35(8): 1381-95.

18. Kanaya AM, Araneta MRG, Pawlowsky SB, Barrett-Connor E, Grady D, Vittinghoff E, et al. Restorative yoga and metabolic risk factors: the Practicing Restorative Yoga vs. Stretching for the Metabolic Syndrome (PRYSMS) randomized trial. J Diabetes Complicat. 2014;28(3):406-12.

19. Friedewald WT, Levy RI, Fredrickson DS. Estimation of the concentration of low-density lipoprotein cholesterol in plasma, without use of the preparative ultracentrifuge. Clin Chem. 1972;18(6):499 502 .

20. Faraldi M, Gomarasca M, Sansoni V, Perego S, Banfi G, Lombardi G. Normalization strategies differently affect circulating miRNA profile associated with the training status. Sci Rep. 2019;9(1):1584.

21. Marabita F, de Candia P, Torri A, Tegnér J, Abrignani S, Rossi RL. Normalization of circulating microRNA expression data obtained by quantitative real-time RT-PCR. Brief Bioinform. 2016;17(2): 204-12.

22. Livak KJ, Schmittgen TD. Analysis of relative gene expression data using real-time quantitative PCR and the 2(-Delta Delta C(T)) Method. Methods. 2001;25(4):402-8.

23. Cui X, You L, Zhu L, Wang X, Zhou Y, Li Y, et al. Change in circulating microRNA profile of obese children indicates future risk of adult diabetes. Metabolism. 2018;78:95-105.

24. Rong Y, Bao W, Shan Z, Liu J, Yu X, Xia S, et al. Increased microRNA-146a levels in plasma of patients with newly diagnosed type 2 diabetes mellitus. PLoS One. 2013;8(9):e73272.

25. Ortega FJ, Mercader JM, Catalán V, Moreno-Navarrete JM, Pueyo $\mathrm{N}$, Sabater M, et al. Targeting the circulating microRNA signature of obesity. Clin Chem. 2013;59(5):781-92.

26. Alipoor B, Ghaedi H, Meshkani R, Torkamandi S, Saffari S, Iranpour M, et al. Association of MiR-146a expression and type 2 diabetes mellitus: a meta-analysis. Int J Mol Cell Med. 2017;6(3): 156-63. 
27. Balasubramanyam M, Aravind S, Gokulakrishnan K, Prabu P, Sathishkumar C, Ranjani H, et al. Impaired miR-146a expression links subclinical inflammation and insulin resistance in type 2 diabetes. Mol Cell Biochem. 2011;351(1-2):197-205.

28. Raitoharju E, Lyytikäinen LP, Levula M, Oksala N, Mennander A, Tarkka M, et al. miR-21, miR-210, miR-34a, and miR-146a/b are up-regulated in human atherosclerotic plaques in the Tampere Vascular Study. Atherosclerosis. 2011;219(1):211-7.

29. Backes C, Kehl T, Stöckel D, Fehlmann T, Schneider L, Meese E, et al. miRPathDB: a new dictionary on microRNAs and target pathways. Nucleic Acids Res. 2017;45(D1):D90-6.

30. Corral-Fernandez NE, et al. Dysregulated miR-155 expression in peripheral blood mononuclear cells from patients with type 2 diabetes. Exp Clin Endocrinol Diabetes. 2013;121(6):347-53.

31. Baldeon RL, et al. Decreased serum level of miR-146a as sign of chronic inflammation in type 2 diabetic patients. PLoS One. 2014;9(12):e115209.

32. Wildman RP, Muntner P, Reynolds K, McGinn A, Rajpathak S, Wylie-Rosett J, et al. The obese without cardiometabolic risk factor clustering and the normal weight with cardiometabolic risk factor clustering: prevalence and correlates of 2 phenotypes among the US population (NHANES 1999-2004). Arch Intern Med. 2008;168(15):1617-24.

33. Xia SF, Duan XM, Cheng XR, Chen LM, Kang YJ, Wang P, et al. Role of miR-383 and miR-146b in different propensities to obesity in male mice. J Endocrinol. 2017;234(2):201-16.

34. Nelson M, Runtsch MC, O'Connell R. Anti-inflammatory miR146 a is protective against diet-induced obesity and regulates macrophage metabolism. J Immunol. 2018;200(1 Supplement):166.51.

35. Roos J, Enlund E, Funcke JB, Tews D, Holzmann K, Debatin KM, et al. miR-146a-mediated suppression of the inflammatory response in human adipocytes. Sci Rep. 2016;6:38339.

36. Sulistyoningrum DC, Gasevic D, Lear SA, Ho J, Mente A, Devlin AM. Total and high molecular weight adiponectin and ethnicspecific differences in adiposity and insulin resistance: a crosssectional study. Cardiovasc Diabetol. 2013;12:170.

37. Mente A, Razak F, Blankenberg S, Vuksan V, Davis AD, Miller R, et al. Ethnic variation in adiponectin and leptin levels and their association with adiposity and insulin resistance. Diabetes Care. 2010;33(7):1629-34.

38. Steppan CM, Bailey ST, Bhat S, Brown EJ, Banerjee RR, Wright $\mathrm{CM}$, et al. The hormone resistin links obesity to diabetes. Nature. 2001;409(6818):307-12.

39. Fluitt MB, Kumari N, Nunlee-Bland G, Nekhai S, Gambhir KK. miRNA-15a, miRNA-15b, and miRNA-499 are Reduced in Erythrocytes of Pre-Diabetic African-American Adults. Jacobs J Diabetes Endocrinol. 2016 Dec;2(1):014. Epub 2016 Nov 15. PMID: 29399662; PMCID: PMC5792081.

40. Jiménez-Lucena R, Rangel-Zúñiga OA, Alcalá-Díaz JF, LópezMoreno J, Roncero-Ramos I, Molina-Abril H, et al. Circulating miRNAs as Predictive Biomarkers of Type 2 Diabetes Mellitus Development in Coronary Heart Disease Patients from the CORDIOPREV Study. Mol Ther Nucleic Acids. 2018 Sep 7;12:
146-157. https://doi.org/10.1016/j.omtn.2018.05.002 Epub 2018 May 8. PMID: 30195754; PMCID: PMC6023857.

41. Liu L, Wang D, Qiu Y, Dong H, Zhan X. Overexpression of microRNA-15 increases the chemosensitivity of colon cancer cells to 5-fluorouracil and oxaliplatin by inhibiting the nuclear factorkappaB signalling pathway and inducing apoptosis. Exp Ther Med. 2018;15(3):2655-60.

42. Zhang H, Li W. microRNA-15 Activates NF-kappaB pathway via down regulating expression of adenosine $\mathrm{A} 2$ receptor in ulcerative colitis. Cell Physiol Biochem. 2018;51(4):1932-44.

43. Liu Y, Gao G, Yang C, Zhou K, Shen B, Liang H, et al. The role of circulating microRNA-126 (miR-126): a novel biomarker for screening prediabetes and newly diagnosed type 2 diabetes mellitus. Int J Mol Sci. 2014;15(6):10567-77.

44. Li Y, Zhou Q, Pei CZ, Liu B, Li MQ, Fang L, et al. Hyperglycemia and advanced glycation end products regulate miR-126 expression in endothelial progenitor cells. J Vasc Res. 2016;53(1-2):94-104.

45. Al-Kafaji G, et al. Circulating endothelium-enriched microRNA126 as a potential biomarker for coronary artery disease in type 2 diabetes mellitus patients. Biomarkers. 2017;22(3-4):268-78.

46. Wu K, Yang Y, Zhong Y, Ammar HM, Zhang P, Guo R, et al. The effects of microvesicles on endothelial progenitor cells are compromised in type 2 diabetic patients via downregulation of the miR126/VEGFR2 pathway. Am J Physiol Endocrinol Metab. 2016;310(10):E828-37.

47. Olivieri F, Spazzafumo L, Bonafè M, Recchioni R, Prattichizzo F, Marcheselli F, et al. MiR-21-5p and miR-126a-3p levels in plasma and circulating angiogenic cells: relationship with type 2 diabetes complications. Oncotarget. 2015;6(34):35372-82.

48. Zhang T, Li L, Shang Q, Lv CF, Wang CY, Su B. Circulating miR126 is a potential biomarker to predict the onset of type 2 diabetes mellitus in susceptible individuals. Biochem Biophys Res Commun. 2015;463(1-2):60-3.

49. Wang X, Sundquist J, Zöller B, Memon AA, Palmér K, Sundquist $\mathrm{K}$, et al. Determination of 14 circulating microRNAs in Swedes and Iraqis with and without diabetes mellitus type 2. PLoS One. 2014;9(1):e86792.

50. Ortega FJ, Mercader JM, Moreno-Navarrete JM, Rovira O, Guerra E, Esteve E, et al. Profiling of circulating microRNAs reveals common microRNAs linked to type 2 diabetes that change with insulin sensitization. Diabetes Care. 2014;37:1375-83.

51. Demirsoy IH, Ertural DY, Balci Ș, Çınkır Ü, Sezer K, Tamer L, et al. Profiles of circulating MiRNAs following metformin treatment in patients with type 2 diabetes. J Med Biochem. 2018;37(4):499-506.

52. Conomos MP, Laurie CA, Stilp AM, Gogarten SM, McHugh CP, Nelson SC, et al. Genetic diversity and association studies in US hispanic/latino populations: applications in the hispanic community health study/study of Latinos. Am J Hum Genet. 2016;98(1):16584.

Publisher's note Springer Nature remains neutral with regard to jurisdictional claims in published maps and institutional affiliations. 